\title{
Evaluation of Metabolic Monitoring and Diabetes Incidence in the First Year for Veterans Newly Started on an Atypical Antipsychotic
}

\author{
Clayton Hamilton, PharmD; Janice Taylor, PharmD, BCBS; and Scott Mambourg, PharmD, BCPS, AAHIVP
}

Purpose: This retrospective chart review is an evaluation of patient and health care provider adherence to a metabolic monitoring protocol as well as progression to type 2 diabetes mellitus (T2DM) in the first year after atypical antipsychotic initiation.

Methods: Patient $(N=1,651)$ data from February 2014 to February 2019 were collected from the US Department of Veterans Affairs (VA) Corporate Data Warehouse for 8 VA medical centers within Veterans Integrated Service Network (VISN) 21. Collected data included patient demographic, laboratory results, and medication history.

Results: In the final cohort, $6 \%$ of patients were found to have progressed to T2DM. Adherence to appropriate metabolic monitoring was found to be overall suboptimal.

Conclusions: The findings of this project demonstrate that patients in VISN 21 who were previously antipsychotic-naïve and nondiabetic may be at increased risk of progression to T2DM when compared with the general population. To effectively manage patient risk, health care providers and patients should improve adherence to metabolic monitoring.
Clayton Hamilton is an Informatics Pharmacist at the George E. Wahlen Medical Center in Salt Lake City, Utah. Janice Taylor is the Veterans Integrated Service Network (VISN) 21 Pharmacy Benefits Management office Program and Data Manager, and Scott Mambourg is the VISN 21 Pharmacy Executive, both in Reno, Nevada. Correspondence: Clayton Hamilton (clayton.hamilton@va.gov)

Fed Pract. 2020;37(11):532-535. doi: 10.12788/fp.0067
A typical antipsychotic use may result in metabolic abnormalities, such as hyperglycemia, dyslipidemia, weight gain, and metabolic syndrome. These adverse effects (AEs) can cause progression to type 2 diabetes mellitus (T2DM) as well as increased risk of cardiovascular disease and cardiac mortality. Individuals diagnosed with T2DM have medical expenses that are about 2.3 times higher than individuals without diabetes. ${ }^{1,2}$ The risk of experiencing metabolic abnormalities is likely elevated for patients who were antipsychotic-naive prior to initiation. ${ }^{3}$

In response to an increased awareness of atypical antipsychotic-related AEs, the American Diabetes Association (ADA) and American Psychiatric Association (APA) released a consensus statement in 2004 with a metabolic monitoring protocol for patients initiating or changing to a new antipsychotic medication. ${ }^{4}$ Within the first year after initiation, the ADA/APA consensus statements recommends that clinicians acquire a personal and family history, weight, body mass index (BMI), waist circumference, blood pressure (BP), fasting plasma glucose, and fasting lipid profile at the initial patient visit. Patient weight is recommended to be collected at 4 weeks and again 8 weeks later. Twelve weeks after the initial visit, weight, BMI, BP, fasting plasma glucose and a fasting lipid profile are recommended to be collected and assessed for abnormalities. Weight is then recommended to be assessed every 3 months thereafter. Review of personal and family history, waist circumference, $\mathrm{BP}$, and a fasting plasma glucose is recommended to occur annually. Finally, a fasting lipid profile is to be collected every 5 years.

Since the initial consensus statement release, metabolic monitoring of patients prescribed antipsychotic medications has been found to be inadequate within several large health care organizations. ${ }^{5,6}$ Mittal and colleagues reviewed metabolic monitoring practices occurring in 32 facilities within the Veterans Health Administration (VHA) and found that monitoring practices in the first 90 days after antipsychotic initiation were largely nonadherent to the ADA/ APA consensus statement recommendations. ${ }^{6}$ Medical staff in Veterans Integrated Service Network 21 (VISN 21) currently serve about 268,000 veterans actively receiving care across California, Nevada, and the Pacific Islands. To support veteran care in the fields of mental health and medication safety, the VISN 21 pharmacy benefits manager office created a clinical dashboard that identifies veterans who are currently prescribed an antipsychotic and have not completed at least 1 annual blood glucose test. While this dashboard is a valuable tool for tracking patient care for those who have been prescribed an antipsychotic $>1$ year, it does not consider the ADA/APA recommendations for more frequent monitoring in the 
TABLE 1 Demographics of Patients Taking Atypical Antipsychotic Medications in Veterans Integrated Service Network 21

\begin{tabular}{lccccc} 
Antipsychotic Medications & Patients, No. & Age, Mean (SD) & Male, No. (\%) & BMI, Mean (SD) & Progressed to T2DM, No. (\%) \\
\hline Quetiapine & 884 & $56(15.1)$ & $793(90)$ & $28.9(7.1)$ & $48(5.4)$ \\
\hline Risperidone & 304 & $55(14.2)$ & $273(90)$ & $29.3(5.6)$ & $25(8.2)$ \\
\hline Aripiprazole & 226 & $54(13.1)$ & $180(80)$ & $30.5(5.7)$ & $11(4.9)$ \\
\hline Olanzapine & 135 & $57(14.8)$ & $123(91)$ & $26.8(4.5)$ & $4(5.2)$ \\
\hline Ziprasidone & 44 & $51(11.6)$ & $32(73)$ & $31.4(6.4)$ & $3(7.5)$ \\
\hline Lurasidone & 40 & $50(14.0)$ & $30(75)$ & $29.6(5.3)$ & 0 \\
\hline Clozapine & 10 & $55(9.9)$ & $10(100)$ & $29.9(2.9)$ & 0 \\
\hline Paliperidone & 7 & $49(17.1)$ & $5(71)$ & $26.0(3.6)$ & $1(100)$ \\
\hline Asenapine & 1 & $38(0.0)$ & $1(100)$ & $39.0(8.5)$ & $\mathbf{9 9}(6.0)$
\end{tabular}

Abbreviations: BMI, body mass index; T2DM, type 2 diabetes mellitus.

first year after initiation. A literature review found no citations of a systematic evaluation of adherence to ADA/APA monitoring recommendations or patient progression to T2DM in the first year after antipsychotic initiation for an antipsychotic-naive veteran population. The goal of this quality improvement project is to assess VHA health care provider and patient adherence to the 2004 consensus statement recommendations within the first year after initiation for previously antipsychotic-naive patients receiving an atypical antipsychotic and determine rate of progression to T2DM.

\section{METHODS}

The project was reviewed by the University of Nevada-Reno Institutional Review Board and determined to be a nonresearch quality improvement project. This was a retrospective chart analysis that included patients receiving their first-ever atypical antipsychotic across 8 US Department of Veterans Affairs (VA) medical centers within VISN 21. Clinical patient data, including prescription, vital sign, and laboratory information, were extracted from the VA Corporate Data Warehouse using transact sequential query language.

Veterans were included in the final cohort if they met the following criteria: aged $\geq 18$ years at antipsychotic initiation, initiated their first-ever atypical antipsychotic within the VHA between February 2014 and February 2019, continued the antipsychotic for $\geq 1$ year, had a medication possession ratio (MPR) $>80 \%$, and had previously established care within VHA as evidenced by having $\geq 1$ primary care or outpatient mental health visit in the 6 months prior to initiation. The MPR is defined as the sum of the day's supply of all dispensed medications in the project time frame divided by the total number of days in the project time frame.

Veterans were excluded if they initiated any other antipsychotic during the first course, had a prior diagnosis of T2DM, had any prior use of antidiabetic medications, or had a hemoglobin $\mathrm{A}_{1 \mathrm{c}}\left(\mathrm{HbA}_{1 \mathrm{c}}\right)>6.4$ in the year prior to initiation.

The primary outcome was completion of all recommended metabolic monitoring time points in the first year after atypical antipsychotic initiation. The secondary outcome was incidence of T2DM as evidenced by either a $\mathrm{HbA}_{\mathrm{lc}}>6.4$ or diagnosis of T2DM entered into the electronic health record. Baseline monitoring for BP, blood glucose, and lipids were considered complete if a data point was collected between 3 months prior and 1 month after atypical antipsychotic 
TABLE 2 Cohort Metabolic Monitoring Results

\begin{tabular}{lcc} 
Parameters & $\begin{array}{c}\text { Time Points, } \\
\text { No. }\end{array}$ & $\begin{array}{c}\text { Patients Receiving Monitoring } \\
\text { in the First Year, No. (\%) }\end{array}$ \\
\hline Blood pressure & 3 & $492(30)$ \\
\hline $\begin{array}{l}\text { Hemoglobin } \mathrm{A}_{1 \mathrm{c}} \text { and/or } \\
\text { blood glucose }\end{array}$ & 3 & $203(12)$ \\
\hline Lipid panel & 2 & $96(6)$ \\
\hline Weight & 7 & $47(3)$ \\
\hline Received all monitoring & 15 & $3(0.2)$
\end{tabular}

initiation. Baseline monitoring for weight was considered complete if a data point was collected between 3 months prior and 2 weeks after initiation. Follow-up monitoring for BP, blood glucose, and lipids were considered completed if a data point was collected at 3 and 12 months (mean, 1 month). Follow-up monitoring for weight was considered completed if collected at 1,2 , and 3 months (mean, 2 weeks) and at 6, 9, and 12 months (mean, 1 month). Waist circumference data and patient and family history are not collected as capturable data points. Therefore, the authors were unable to include these in the final data extraction.

\section{RESULTS}

The final cohort consisted of 1,651 veterans who met the inclusion criteria. Overall, at antipsychotic initiation the cohort had a mean (SD) age of 55 (14.6) years, was largely male $(88 \%)$, and was considered overweight with a mean (SD) BMI of 29.1 (6.4) (Table 1).

Appropriate BP monitoring was completed most often with 492 patients (30\%) meeting ADA/APA recommendations followed by $\mathrm{HbA}_{1 \mathrm{c}}$ and/or blood glucose monitoring with 203 patients (12\%) completing all time points. Recommended lipid monitoring was completed by 96 patients (6\%). Weight monitoring was completed least often with 47 patients (3\%) completing all recommended time points. Regarding completion of all metabolic monitoring time points, $3(0.2 \%)$ patients in the final cohort were found to have completed all recommended monitoring. Ninety-nine patients (6\%) were found to have progressed to T2DM as indicated by an $\mathrm{HbA}_{1 \mathrm{c}}>6.4$ and/ or entry of a T2DM ninth or tenth edition
International Statistical Classification of Diseases code into the chart (Table 2).

\section{DISCUSSION}

No previous literature exists that reviews adherence to recommended metabolic monitoring guidance up to 1 year after antipsychotic initiation in a previously antipsychotic-naïve cohort within the VHA. Metabolic monitoring was overall incomplete with $0.2 \%$ of the cohort completing all recommended monitoring time points. Weight was the parameter that was least completed. Based on these findings, the authors concluded that efforts are needed to improve completion rates of atypical antipsychotic metabolic monitoring. In the final cohort, $6 \%$ of patients were noted to have progressed to T2DM in the first year after atypical antipsychotic initiation. The actual number of patients progressing to T2DM may be larger because not all received adequate blood glucose monitoring. For comparison, the Centers for Disease Control and Prevention released information in 2015 that stated that the US population has an annual T2DM incidence of about $1 \%$ for adults aged 45 to 64 years. ${ }^{7}$

We understand that individuals with mental health disorders are at increased risk of T2DM compared with that of the general population and hope that this comparison only serves to drive home the point that appropriate metabolic monitoring is vital for this subgroup. The strengths of this project include identification of an area for improvement and encouraging evidence-based monitoring. Utilization of clinical data is a cost-effective and efficient method to improve patient care.

\section{Limitations}

Limitations of this study include the data's dependence on accuracy of entry by the end-user and a lack of available data regarding prescriptions dispensed outside of the VHA. Vital signs data may have been entered into patient notes and not documented in the vitals section of the current medical record causing the appearance of missing data. Access to VHA health services and patient adherence to follow-up appointments were not assessed in this project and could affect patient ability to complete follow-up. The 
final analysis included only patients who remained on 1 atypical antipsychotic for a year and were considered adherent with an MPR $>80 \%$ and did not consider less adherent patients. It is also possible that health care providers who closely monitor metabolic parameters after atypical antipsychotic initiation more frequently switch patients to an alternative atypical antipsychotic while others who monitor less also switch medications less frequently. This could lead to selection of patients with health care providers who are less adherent to metabolic monitoring recommendations.

\section{CONCLUSIONS}

As a result of this study, in VISN 21 several strategies will be implemented to improve monitoring. First, the results of this project will be shared with the subject matter experts of the VISN 21 Mental Health Task Force. This task force serves as a venue for clinicians to meet virtually, discuss clinical topics, as well as to create and distribute strategies to improve patient care. Clinicians at this forum will be encouraged to implement monitoring protocols into routine practice, share best practices with colleagues, and increase patient awareness about the importance of metabolic monitoring. Second, modifications may be applied to the electronic health record to guide metabolic monitoring order entry at the time of prescription entry, which includes development of clinical reminders and laboratory order sets. Third, the clinical data manager team may be leveraged to create an electronic report identifying patients currently receiving suboptimal monitoring in the first year after antipsychotic initiation. The patients identified in this report will be discussed at the recurring VISN 21 Mental Health Task Force meeting, and strong practices will be shared with the medical centers across VISN 21. Other strategies under consideration include requiring proof of metabolic monitoring completion prior to allowing further atypical antipsychotic refills and providing direct provider education regarding the ADA/APA metabolic monitoring recommendations via the academic detailing service in effort to standardize clinical care.

\section{Author disclosures}

The authors report no actual or potential conflicts of interest with regard to this article.

\section{Disclaimer}

The opinions expressed herein are those of the authors and do not necessarily reflect those of Federal Practitioner, Frontline Medical Communications Inc., the US Government, or any of its agencies. This article may discuss unlabeled or investigational use of certain drugs. Please review the complete prescribing information for specific drugs or drug combinations-including indications, contraindications, warnings, and adverse effects-before administering pharmacologic therapy to patients.

\section{References}

1. Goff DC, Sullivan LM, McEvoy JP, et al. A comparison of ten-year cardiac risk estimates in schizophrenia patients from the CATIE study and matched controls. Schizophr Res. 2005;80(1):45-53. doi:10.1016/j.schres.2005.08.010

2. American Diabetes Association. Economic costs of diabetes in the U.S. in 2012. Diabetes Care. 2013;36(4):1033-1046. doi:10.2337/dc12-2625

3. Rajkumar AP, Horsdal HT, Wimberley T, et al. Endogenous and antipsychotic-related risks for diabetes mellitus in young people with schizophrenia: a Danish populationbased cohort study. Am J Psychiatry. 2017;174(7):686-694. doi:10.1176/appi.ajp.2016.16040442

4. American Diabetes Association; American Psychiatric Association; American Association of Clinical Endocrinologists; North American Association for the Study of Obesity. Consensus development conference on antipsychotic drugs and obesity and diabetes. Diabetes Care. 2004;27(2):596-601. doi:10.2337/diacare.27.2.596

5. Morrato EH, Druss B, Hartung DM, et al. Metabolic testing rates in 3 state Medicaid programs after FDA warnings and ADA/APA recommendations for second-generation antipsychotic drugs. Arch Gen Psychiatry. 2010;67(1):17-24. doi:10.1001/archgenpsychiatry.2009.179

6. Mittal D, Li C, Williams JS, Viverito K, Landes RD, Owen RR. Monitoring veterans for metabolic side effects when prescribing antipsychotics. Psychiatr Serv. 2013;64(1):28-35. doi:10.1176/appi.ps.201100445

7. Centers for Disease Control and Prevention. National diabetes statistics report, 2020. https://www.cdc.gov /diabetes/data/statistics-report/index.html. Updated August 28, 2020. Accessed October 9, 2020. 